\title{
Association of Leukotriene Gene Variants and Plasma LTB4 Levels with Coronary Artery Disease in Asian Indians
}

\author{
Jiny Nair, ${ }^{1}$ Jayashree Shanker, ${ }^{1}$ Prathima Arvind, ${ }^{1}$ \\ Srikarthika Jambunathan, ${ }^{1}$ and Vijay V. Kakkar ${ }^{2,3}$ \\ ${ }^{1}$ Mary and Garry Weston Functional Genomics Unit, Thrombosis Research Institute, Narayana Hrudayalaya 258/A, Bommasandra \\ Industrial Area, Anekal Taluk, Bangalore, Karnataka 560099, India \\ ${ }^{2}$ Thrombosis Research Institute, Bangalore 560099, India \\ ${ }^{3}$ Thrombosis Research Institute, London, SW3 6LR, UK
}

Correspondence should be addressed to Jayashree Shanker; jayashreeshanker@triindia.org.in

Received 24 April 2013; Accepted 14 May 2013

Academic Editors: D. Guidolin, A. Mugge, B. Tesfamariam, and C.-C. Wu

Copyright (C) 2013 Jiny Nair et al. This is an open access article distributed under the Creative Commons Attribution License, which permits unrestricted use, distribution, and reproduction in any medium, provided the original work is properly cited.

\begin{abstract}
Leukotrienes are potent inflammatory and lipid mediators that participate in atherosclerosis. We analyzed the association of Leukotriene gene (ALOX5, ALOX5AP, LTA4H, and LTC4S) polymorphisms and plasma Leukotriene B4 (LTB4) levels with coronary artery disease (CAD) in a representative cohort of Asian Indians. In all, 136 functional single nucleotide polymorphisms (SNPs) were selected using in silico tools. Forty-five polymorphic SNPs were ranked for predicted functional effect using FastSNP. Finally, 14 functional SNPs along with 10 SNPs identified from the literature were genotyped in 340 CAD patients and 340 controls. Plasma LTB4 levels were measured in 150 cases and 150 controls. None of the 24 SNPs showed significant association with CAD. Plasma LTB4 levels were higher in cases than in controls $(76.42 \pm 4.46 \mathrm{pg} / \mathrm{mL}$ versus $60.89 \pm 2.61 \mathrm{pg} / \mathrm{mL})(P=0.003)$, with greater risk being associated with the top quartile as compared to the bottom quartile after adjusting for potential confounders (OR 8.94, 95\% CI 2.56-31.95; $P=0.001)$. Four SNPs in the LTA4H gene showed significant association with LTB4 levels $(P<0.05)$ of which rs1978331 $(P=0.035)$ remained significant after correction for multiple testing. LTB4 showed strong correlation with lipids $(r=0.24-34)$ only in cases. Our pilot study suggests that the association between Leukotrienes gene polymorphisms and CAD risk may be modulated through plasma LTB4 levels.
\end{abstract}

\section{Introduction}

Both genetics and environmental factors combined with unhealthy lifestyle play an important role in the development of coronary artery disease (CAD) [1]. Hypercholesterolemia and inflammation are two key players in CAD [2]. Leukotrienes act as mediators in the lipid and inflammatory pathway and are synthesized from Arachidonic acid through a cascade process in immune-competent cells. During this process, 5-lipoxygenase (ALOX5) in association with 5LO activating protein (ALOX5AP), metabolizes Arachidonic acid to Leukotriene A4 (LTA4). LTA4 is unstable and is further converted to Leukotriene B4 (LTB4) and Cysteinyl Leukotrienes (LTC4, LTD4, and LTE4) by LTA4 Hydrolase (LTA4H) and LTC4 Synthase (LTC4S) enzymes, respectively [3]. These Leukotrienes then modulate immune response by triggering the synthesis and release of cytokines and interacting with their cognate receptors [4].

Leukotrienes are known to play a significant role in CAD development, based on biochemical, genetic, and pharmacological studies [5-7]. Immunohistochemical studies demonstrate abundant expression of Leukotrienes on the arterial walls $[8,9]$, which correlate with symptoms of plaque instability [10]. Population studies have also explored the association of Leukotriene gene polymorphisms with cardiovascular disease. The deCODE Genetics group identified two distinct haplotypes, Hap A and Hap B in the ALOX5AP gene through linkage study, that were associated with elevated risk of myocardial infarction (MI) and stroke in two independent cohorts. Further, functional studies provided evidence that these variants induce excess production of LTB4 $[11,12]$. The 
same group also reported modest increase in the risk of MI in carriers of haplotype, Hap $\mathrm{K}$ in the LTA4H gene [13]. In addition, there are reports of ALOX5 promoter variants containing multiple Spl binding sites that are associated with higher risk of MI $[14,15]$ and promoter variant rs730012 of LTC4S gene which is associated with greater risk of early carotid atherosclerosis [16].

Despite these significant findings, many other studies do not concur with these reports [17-19]. Such inconsistent reports on different ethnic populations tend to create ambiguity on the direct role of Leukotriene genetic variants in the pathogenesis of atherosclerosis. A noteworthy aspect is that most studies have been carried out on Caucasians while, to our knowledge, there are no studies relating to Leukotrienes and CAD on Asian Indians. Given the high rate of morbidity and mortality compounded by premature disease onset in Asian Indians, there is an urgent need to identify novel genes and other predisposing factors that contribute to higher disease prevalence in this population. In addition, the most frequently studied haplotype, Hap A studied by Helgadottir et al., included SNPs located outside the region of the gene while Hap B was constructed using SNPs without known functional effects. This triggered our interest to screen for functional variants in these genes. The aim of this pilot case-control study was therefore to evaluate the genetic contribution of Leukotriene variants with CAD and to test the association of these variants with plasma LTB4 levels in a representative cohort of Asian Indians.

\section{Materials and Methods}

2.1. Study Subjects. A total of 680 participants comprising of 340 angiographically confirmed CAD patients (cases) and 340 age and gender matched controls were selected from the ongoing Indian Atherosclerosis Research Study (IARS). An overview of the IARS study design has been previously described [20]. Briefly, it is an ongoing epidemiological study with an objective to investigate the genetic factors and biomarkers against a backdrop of conventional cardiovascular risk factors in Asian Indians living in India. Recruitment of cases and controls was based on predefined inclusion/exclusion criteria. Inclusion criteria for cases were as follows: angiographically confirmed presence of CAD with $>70 \%$ stenosis in any major epicardial artery, diagnosis or $>50 \%$ stenosis in two or more arteries, patients undergoing percutaneous transluminal coronary angioplasty or bypass graft surgery, and age at onset $\leq 60$ years for men and $\leq 65$ years for women. Control subjects were clinically asymptomatic for $\mathrm{CAD}$, enrolled from the same geographical area as that of the proband, did not have a family history of cardiovascular disease and showed normal electrocardiogram readings. None of the participants had any concomitant infection. Detailed demographics, anthropometrics, vital parameters, medical history, medication, and pedigree information were recorded for each participant through personal interview. Prevalence of type 2 diabetes, hypertension, and cardiovascular disease was ascertained based on self-report of physician's diagnosis and/or use of prescription medications along with perusal of their medical records. Body mass index was calculated as a ratio of weight in $\mathrm{kg}$ to height in meter ${ }^{2}$. All participants provided signed, voluntary informed consent and the study was approved by the Ethics Committee of TRI and follows the guidelines of the Indian Council of Medical Research (ICMR) on bioethics [21].

2.2. Clinical Laboratory Measurements. Plasma levels of total cholesterol (TC), triglyceride (TG), and HDL cholesterol were measured using reagents from Siemens Dimension Flex reagent cartridge (Siemens Healthcare Diagnostics Ltd, UK) and standards from Randox laboratories (Crumlin, UK) using Siemens dimension Xpand plus instrument (Siemens Dade Behring, Liederbach, Germany). Liquicheck lipid control was used as an assayed quality control (Bio-Rad) that was run along with every experiment. Low-density lipoproteincholesterol (LDL-c) was calculated using the Friedwald's formula [22].

2.3. SNP Selection Process. Figure 1 provides a schematic overview of the SNP selection process. We selected a total of 136 functional variants belonging to four genes, ALOX5, ALOX5AP, LTA4H, and LTC4S from the Leukotriene pathway using Genomatix:SNP analysis (http://www.genomatix.de) and SNPinfo in silico tools, which pick up SNPs that are more likely to affect the biological function [23]. SNPs located in the coding region, transcription-factor-binding sites (TFBS), splice sites, microRNA-binding sites, and untranslated regions (UTRs) were selected using SNPinfo, while Genomatix was used to select SNPs affecting the TFBS and protein sequence. SNPs generated by both tools were combined to compile the final list of 136 functional SNPs (Table S1) (see Supplementary Materials available online at http://dx.doi.org/10.1155/2013/985743). These SNPs were further short listed based on their polymorphic status and risk scores using FastSNP [24], which resulted in the final selection of 14 functional SNPs with low-medium risk scores for genotyping and located in the $5^{\prime}$ or $3^{\prime}$ region (Table 1$)$. The FastSNP prioritization result is based on predicted functional effects and their estimated risk.

With regard to the literature-based SNPs, we selected Hap A and Hap B blocks of ALOX5AP and Hap K block of LTA4H $[12,13]$, and the haplotypes were defined by the following SNPs, Hap A: rs17222814 (G), rs10507391 (T), rs4769874 (G), rs9551963 (A); Hap B: rs17216473 (A), rs10507391 (A), rs9315050 (A), $\operatorname{rs17222842}(\mathrm{G})$; Hap K: rs61937881 (C), rs2660880 (G), rs6538697 (T), rs1978331 (A), rs17677715 (T), rs2247570 (T), rs2660898 (T), rs2540482 (C), rs2660845 (G), and $\operatorname{rs} 2540475(\mathrm{G})$. To minimize the number of SNPs required to be genotyped, tagging SNPs were selected based on the linkage disequilibrium (LD) distribution pattern between the functional and literature based SNPs. Finally, 10 additional SNPs from literature were selected from ALOX5AP (Hap A-rs17222814, Hap B-rs17216473, rs9315050, and rs17222842) and LTA4H (rs2540475, rs2247570, rs17677715, rs6538697, rs2660880, and rs61937881) genes, respectively. 


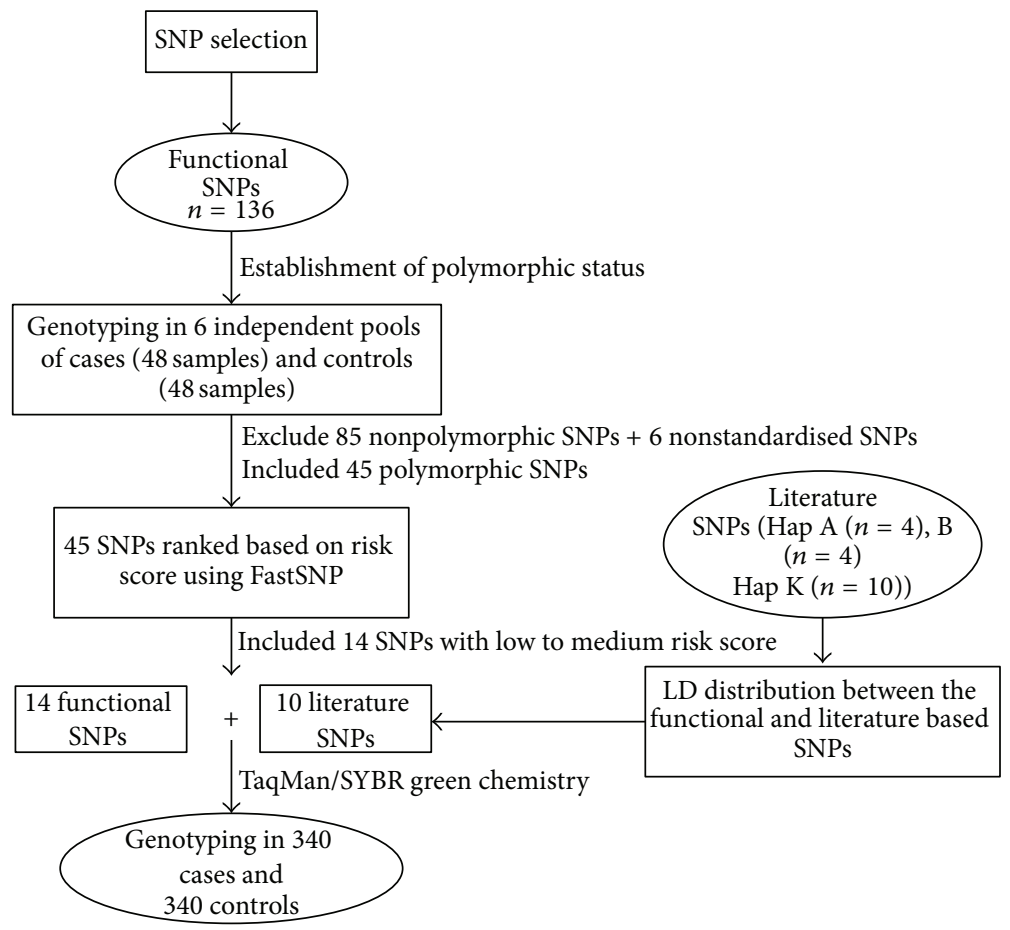

FIGURE 1: Flow chart showing the SNP selection process.

TABLE 1: Final list of functional SNPs $(N=14)$ selected for genotyping based on the predicted functional effect and estimated risk score.

\begin{tabular}{|c|c|c|c|c|c|}
\hline Gene & rs ID & Position (bp) & Type of SNP & Fast SNP rank & Literature Tagging SNP \\
\hline \multirow{4}{*}{ ALOX5 } & rs2291427 & 45256230 & intronic & $(1-2)$ & \\
\hline & rs12762303 & 45189177 & $5^{\prime}$ upstream & $(1-3)$ & \\
\hline & rs1864414 & 45190567 & Intronic & $(1-2)$ & \\
\hline & rs 41314980 & 45261505 & $3^{\prime}$ UTR & $(0-0)$ & \\
\hline \multirow{5}{*}{ ALOX5AP } & rs4769055 & 30207830 & intronic & $(1-2)$ & rs10507391 (Hap A/B) \\
\hline & rs17222919 & 30206329 & $5^{\prime}$ upstream & $(0-0)$ & \\
\hline & rs4360791 & 30216020 & intronic & $(0-0)$ & rs9551963 (Hap A) \\
\hline & rs3803277 & 30216308 & intronic & $(0-0)$ & rs9551963 (Hap A) \\
\hline & rs1132340 & 30236462 & $3^{\prime}$ UTR & $(0-0)$ & rs4769874 (Hap A) \\
\hline \multirow{4}{*}{ LTA4H } & rs2660899 & 94954551 & $5^{\prime}$ upstream & $(1-3)$ & \\
\hline & rs17525495 & 94953508 & $5^{\prime}$ upstream & $(0-0)$ & \\
\hline & rs2660898 & 94950128 & intronic & $(1-2)$ & Hap K \\
\hline & rs1978331 & 94933332 & intronic & $(1-2)$ & Hap K, rs2540482, rs2660845 \\
\hline LTC4S & rs730012 & 179153244 & $5^{\prime}$ upstream & $(0-0)$ & \\
\hline
\end{tabular}

1-2: very low to low; $1-3$ : very low to medium; 0: unknown.

2.4. SNP Genotyping. Genomic DNA was extracted using a modified salting out procedure [25] and quantitated on ND1000 spectrophotometer (NanoDrop Technologies Inc., Wilmington, DE, USA). Primers for the 14 SNPs were designed using RExPrimer, an integrated graphical webbased method (http://www4a.biotec.or.th/rexprimer), and the oligonucleotides were purchased from Sigma-Aldrich (St. Louis, USA). Forty-six primer pairs were designed to annotate for 136 SNPs (Table S2) and their polymorphic status was established on pooled DNA samples [26], based on targeted sequencing. Genomic DNA from 8 randomly selected individuals was pooled in equal volumes at $20 \mathrm{ng} / \mu \mathrm{L}$ final concentration to construct a single DNA pool. In total, 6 DNA pools for cases and controls were constructed separately from 48 cases and 48 controls, respectively. Regions of interest were amplified on a 9700 GeneAmp PCR instrument and the products were sequenced bi-directionally using Big Dye Terminator v3.1 sequencing kit from ABI as per manufacturer's recommendations and analyzed with 3130XL automated genetic analyzer (Applied Biosystems, Foster City, USA) using the SeqScape v 2.5 software (Applied Biosystems, Foster City, USA). 
TABLE 2: Clinical characteristics of the study population.

\begin{tabular}{|c|c|c|c|}
\hline & Controls $(n=340)$ & Cases $(n=340)$ & $P$ value \\
\hline Age (in years) & $47.39 \pm 6.66$ & $46.93 \pm 6.68$ & 0.368 \\
\hline Male $n(\%) /$ female $n(\%)$ & $250(73.5) / 90(26.5)$ & $246(73) / 91(27)$ & \\
\hline $\operatorname{BMI}\left(\mathrm{kg} / \mathrm{m}^{2}\right)$ & $25.74 \pm 4.74$ & $25.97 \pm 4.48$ & 0.517 \\
\hline Total cholesterol (mg/dL) & $172.74 \pm 39.71$ & $139.83 \pm 39.24$ & $<0.0001$ \\
\hline HDL-cholesterol (mg/dL) & $41.44 \pm 9.78$ & $34.8 \pm 9.95$ & $<0.0001$ \\
\hline LDL-cholesterol (mg/dL) & $99.88 \pm 31.05$ & $72.52 \pm 32.45$ & $<0.0001$ \\
\hline Triglycerides (mg/dL) & $162.11 \pm 112.47$ & $166.51 \pm 83.07$ & $<0.0001$ \\
\hline Hypertension $n(\%)$ & $49(14.4)$ & $169(50.3)$ & $<0.0001$ \\
\hline Diabetes $n(\%)$ & $54(15.9)$ & $137(40.8)$ & $<0.0001$ \\
\hline SBP (mmHg) & $124.2 \pm 16.66$ & $119.9 \pm 15.28$ & $<0.0001$ \\
\hline $\mathrm{DBP}(\mathrm{mmHg})$ & $81.63 \pm 10.75$ & $78.15 \pm 9.04$ & $<0.0001$ \\
\hline Age at onset of CAD & $\ldots$ & $45.77 \pm 7.03$ & $\ldots$ \\
\hline Positive family history & $\ldots$ & $225(66.2)$ & $\ldots$ \\
\hline Negative family history & $\ldots$ & $115(33.8)$ & $\ldots$ \\
\hline Stable angina & $\ldots$ & $147(44.5)$ & $\ldots$ \\
\hline Unstable angina & $\ldots$ & $179(54.2)$ & $\ldots$ \\
\hline \multicolumn{4}{|c|}{ Number of disease vessels $n(\%)$} \\
\hline 1 & & $59(18.4)$ & $\ldots$ \\
\hline 2 & & $83(25.9)$ & $\ldots$ \\
\hline 3 & & $179(55.8)$ & $\ldots$ \\
\hline
\end{tabular}

Continuous variables are expressed as mean \pm standard deviation. BMI: body mass index; HDL-c: high density lipoprotein cholesterol; LDL-c: low density lipoprotein cholesterol; SBP: systolic blood pressure; DBP: diastolic blood pressure.

After establishing the polymorphic status of SNPs followed by ranking, 14 functional SNPs were genotyped in 680 subjects using allele specific melting temperature Tm shift method [27]. The two allele specific forward primers and one common reverse primer were designed using Primer Express V2.0 (Applied Biosystems, Foster City, CA, USA). The primer sequence is shown in Table S3. Genotyping of 10 literature-based SNPs for 680 subjects was performed by TaqMan probe-based assay. Tm-shift genotyping and allelic discrimination analysis was performed on ABI $7900 \mathrm{HT}$ Sequence Detection System using SDSv2.1 software (Applied Biosystems, Foster City, CA, USA). Ten percent of samples were analyzed in duplicates to confirm genotype accuracy and further verified by sequencing. We obtained $99.5 \%$ accuracy in the genotype call rates.

2.5. LTB4 Immunoassay. LTB4 immunoassay was performed in EDTA plasma samples of 150 cases and 150 matched controls using ELISA kit (Cayman Chemical Company, Ann Arbor, MI, USA) according to the manufacturer's instructions. Absorbance was read at $412 \mathrm{~nm}$ with Bio-Tek power waveX microplate reader (Bio-Tek instrument, Vermont, USA). The intraassay and interassay variability were less than $10 \%$, with $100 \%$ specificity, and $13 \mathrm{pg} / \mathrm{mL}$ assay detection limits.

2.6. Statistical Analysis. All variants were tested for HardyWeinberg equilibrium (HWE) in the control group. SNPs deviating from $\operatorname{HWE}(P<0.05)$ were excluded from further analysis. HWE, odds ratio (ORs) at 95\% confidence intervals
(CIs), haplotype frequency, and corresponding $P$ values for each SNP were estimated separately for cases and controls using SNPstat tool (http://bioinfo.iconcologia.net/index.php?module=Snpstats). Student's $t$-test, ANOVA, and univariate analysis were used to compare the means for continuous variable while $\chi^{2}$ test was used to estimate the association of categorical variables, namely, genotypes with CAD. Normality distribution of plasma LTB4 levels was assessed using Q-Q plot and outliers were removed from further analysis. The final data was log transformed before performing logistic regression to estimate the association between LTB4 quartiles and CAD. Age, gender, hypertension, diabetes, TC, and HDL-c were used as covariates and appropriately adjusted for during the analysis. An interactive linkage disequilibrium (LD) block between SNPs was created using Haploview version 4.2 (http://www.broadinstitute.org/haploview/haploview). Routine analysis was performed using SPSS v17.0 (SPSS Inc., IL, USA). Data were represented as mean \pm standard error of mean, unless otherwise indicated. A nominal $P$ value of $\leq$ 0.05 less was regarded as statistically significant.

\section{Results}

3.1. Clinical Characteristics of Study Subjects. The clinical profile of the study cohort is summarized in Table 2. Out of 340 cases, $225(66.2 \%)$ had positive family history of CVD with at least one affected first degree relative, while 115 subjects (33.8\%) did not have a family history of CVD. Average age was $46.93 \pm 6.68$ years for cases and $47.39 \pm 6.66$ 
TABLE 3: Distribution of genotypes of the short-listed SNP panel in cases and controls and estimated association with risk of CAD.

\begin{tabular}{|c|c|c|c|c|c|}
\hline \multirow{2}{*}{ SNPs } & \multirow{2}{*}{ Genotype } & \multicolumn{2}{|c|}{ Frequency (number of samples) } & \multirow{2}{*}{ OR (95\% CI) } & \multirow{2}{*}{$P$ value } \\
\hline & & Control $(n \%)$ & Cases $(n \%)$ & & \\
\hline \multicolumn{6}{|c|}{ ALOX5 } \\
\hline \multirow{3}{*}{ rs12762303 } & $\mathrm{TT}$ & $210(61.8 \%)$ & $211(62.4 \%)$ & & \\
\hline & $\mathrm{TC}$ & $110(32.4 \%)$ & $113(33.4 \%)$ & $1.01(0.73-1.40)$ & 0.58 \\
\hline & $\mathrm{CC}$ & $20(5.9 \%)$ & $14(4.1 \%)$ & $0.70(0.34-1.42)$ & \\
\hline \multirow{3}{*}{ rs2291427 } & GG & $118(34.7 \%)$ & $131(38.8 \%)$ & & \\
\hline & GA & $169(49.7 \%)$ & $155(45.9 \%)$ & $0.83(0.60-1.16)$ & 0.53 \\
\hline & AA & $53(15.6 \%)$ & $52(15.4 \%)$ & $0.89(0.56-1.41)$ & \\
\hline \multirow{3}{*}{ rs1864414 } & AA & $212(62.4 \%)$ & $217(64.2 \%)$ & & \\
\hline & $\mathrm{AC}$ & $112(32.9 \%)$ & $109(32.2 \%)$ & $0.94(0.68-1.30)$ & 0.72 \\
\hline & $\mathrm{CC}$ & $16(4.7 \%)$ & $12(3.5 \%)$ & $0.73(0.34-1.59)$ & \\
\hline \multirow{3}{*}{ rs41314980 } & $\mathrm{CC}$ & $321(94.4 \%)$ & $318(94.4 \%)$ & & \\
\hline & $\mathrm{CA}$ & $19(5.6 \%)$ & $19(5.6 \%)$ & $1.01(0.52-1.94)$ & 0.98 \\
\hline & AA & $\ldots$ & $\ldots$ & & \\
\hline \multicolumn{6}{|c|}{ ALOX5AP } \\
\hline \multirow{3}{*}{ rs9315050 } & AA & $257(75.6 \%)$ & $258(76.8 \%)$ & & \\
\hline & AG & $78(22.9 \%)$ & 73 (21.7\%) & $0.93(0.65-1.34)$ & 0.93 \\
\hline & GG & $5(1.5 \%)$ & $5(1.5 \%)$ & $1.00(0.28-3.48)$ & \\
\hline \multirow{3}{*}{ rs17216473 } & GG & $212(62.4 \%)$ & $208(61.7 \%)$ & & \\
\hline & GA & $115(33.8 \%)$ & $107(31.8 \%)$ & $0.95(0.68-1.31)$ & 0.27 \\
\hline & AA & $13(3.8 \%)$ & $22(6.5 \%)$ & $1.72(0.85-3.51)$ & \\
\hline \multirow{3}{*}{ rs17222814 } & GG & $280(82.3 \%)$ & $282(83.7 \%)$ & & \\
\hline & GA & $60(17.6 \%)$ & $52(15.4 \%)$ & $0.86(0.57-1.29)$ & 0.09 \\
\hline & AA & $0(0 \%)$ & $3(0.9 \%)$ & $\ldots$ & \\
\hline \multirow{3}{*}{ rs17222842 } & GG & $295(86.8 \%)$ & 307 (91.1\%) & & \\
\hline & GA & 43 (12.7\%) & $30(8.9 \%)$ & $0.67(0.41-1.10)$ & 0.07 \\
\hline & AA & $2(0.6 \%)$ & $0(0 \%)$ & $\ldots$ & \\
\hline \multirow{3}{*}{ rs17222919 } & $\mathrm{TT}$ & 207 (60.9\%) & $204(60.4 \%)$ & & \\
\hline & TG & $116(34.1 \%)$ & $116(34.3 \%)$ & $1.02(0.74-1.41)$ & 0.98 \\
\hline & GG & 17 (5\%) & $18(5.3 \%)$ & $1.08(0.54-2.15)$ & \\
\hline \multirow{3}{*}{ rs4769055 } & $\mathrm{CC}$ & 95 (27.9\%) & $85(25.3 \%)$ & & \\
\hline & CA & $163(47.9 \%)$ & $157(46.7 \%)$ & $1.10(0.77-1.59)$ & 0.48 \\
\hline & AA & 95 (27.9\%) & $85(25.3 \%)$ & $1.30(0.85-1.97)$ & \\
\hline \multirow{3}{*}{ rs4360791 } & AA & $109(32.1 \%)$ & $110(32.5 \%)$ & & \\
\hline & AG & $182(53.5 \%)$ & $168(49.7 \%)$ & $0.91(0.65-1.28)$ & 0.48 \\
\hline & GG & 49 (14.4\%) & $59(17.5 \%)$ & $1.19(0.75-1.89)$ & \\
\hline \multirow{3}{*}{ rs3803277 } & AA & $109(32.1 \%)$ & $117(34.6 \%)$ & & \\
\hline & $\mathrm{AC}$ & $181(53.2 \%)$ & $162(47.9 \%)$ & $0.83(0.60-1.17)$ & 0.38 \\
\hline & CC & $50(14.7 \%)$ & $58(17.2 \%)$ & $1.08(0.68-1.71)$ & \\
\hline \multirow{3}{*}{ rs1132340 } & AA & $261(76.8 \%)$ & $261(77.5 \%)$ & & \\
\hline & AG & 75 (22.1\%) & $72(21.4 \%)$ & $0.96(0.67-1.39)$ & 0.98 \\
\hline & GG & $4(1.2 \%)$ & $4(1.2 \%)$ & $1.00(0.25-4.06)$ & \\
\hline \multicolumn{6}{|c|}{ LTA4H } \\
\hline & TT & $280(82.6 \%)$ & $281(84.1 \%)$ & & \\
\hline rs17677715 & TC & $55(16.2 \%)$ & $51(15.3 \%)$ & $0.92(0.61-1.40)$ & 0.67 \\
\hline & $\mathrm{CC}$ & $4(1.2 \%)$ & $2(0.6 \%)$ & $0.50(0.09-2.74)$ & \\
\hline & AA & $169(49.7 \%)$ & $175(52.1 \%)$ & & \\
\hline rs2540475 & GA & $140(41.2 \%)$ & $135(40.2 \%)$ & $0.93(0.68-1.28)$ & 0.74 \\
\hline & GG & $31(9.1 \%)$ & $26(7.7 \%)$ & $0.81(0.46-1.42)$ & \\
\hline
\end{tabular}


TABLE 3: Continued.

\begin{tabular}{|c|c|c|c|c|c|}
\hline \multirow{2}{*}{ SNPs } & \multirow{2}{*}{ Genotype } & \multicolumn{2}{|c|}{ Frequency (number of samples) } & \multirow{2}{*}{ OR $(95 \% \mathrm{CI})$} & \multirow{2}{*}{$P$ value } \\
\hline & & Control $(n \%)$ & Cases $(n \%)$ & & \\
\hline \multirow{3}{*}{ rs 2247570} & AA & $233(68.5 \%)$ & $244(72.4 \%)$ & & \\
\hline & AG & $97(28.5 \%)$ & $87(25.8 \%)$ & $0.86(0.61-1.20)$ & 0.41 \\
\hline & GG & $10(2.9 \%)$ & $6(1.8 \%)$ & $0.57(0.21-1.60)$ & \\
\hline \multirow{3}{*}{ rs6538697 } & $\mathrm{TT}$ & $205(60.3 \%)$ & $204(60.5 \%)$ & & \\
\hline & $\mathrm{TC}$ & $115(33.8 \%)$ & $117(34.7 \%)$ & $1.02(0.74-1.41)$ & 0.8 \\
\hline & $\mathrm{CC}$ & $20(5.9 \%)$ & $16(4.8 \%)$ & $0.80(0.41-1.60)$ & \\
\hline \multirow{3}{*}{ rs61937881 } & GG & $263(77.3 \%)$ & $263(78 \%)$ & & \\
\hline & GA & $71(20.9 \%)$ & $71(21.1 \%)$ & $1.00(0.69-1.45)$ & 0.60 \\
\hline & AA & $6(1.8 \%)$ & $3(0.9 \%)$ & $0.50(0.12-2.02)$ & \\
\hline \multirow{3}{*}{ rs2660898 } & $\mathrm{TT}$ & $140(41.2 \%)$ & $140(41.5 \%)$ & & \\
\hline & TG & $155(45.6 \%)$ & $160(47.3 \%)$ & $1.03(0.75-1.42)$ & 0.65 \\
\hline & GG & $45(13.2 \%)$ & $37(11 \%)$ & $0.82(0.50-1.35)$ & \\
\hline \multirow{3}{*}{ rs2660899 } & GG & $189(55.6 \%)$ & $183(54.1 \%)$ & & \\
\hline & GT & $123(36.2 \%)$ & $126(37.3 \%)$ & $1.06(0.77-1.47)$ & 0.92 \\
\hline & $\mathrm{TT}$ & $28(8.2 \%)$ & $29(8.6 \%)$ & $1.08(0.62-1.88)$ & \\
\hline \multirow{3}{*}{ rs17525495 } & $\mathrm{CC}$ & $204(60.2 \%)$ & $202(59.8 \%)$ & & \\
\hline & $\mathrm{CT}$ & $116(34.1 \%)$ & $119(35.2 \%)$ & $1.04(0.75-1.43)$ & 0.79 \\
\hline & $\mathrm{TT}$ & $20(5.9 \%)$ & $16(4.8 \%)$ & $0.81(0.41-1.60)$ & \\
\hline \multirow{3}{*}{ rs1978331 } & $\mathrm{TT}$ & $96(28.2 \%)$ & $101(30 \%)$ & & \\
\hline & TC & $172(50.6 \%)$ & $170(50.5 \%)$ & $0.94(0.66-1.33)$ & 0.79 \\
\hline & $\mathrm{CC}$ & $72(21.2 \%)$ & $65(19.4 \%)$ & $0.86(0.55-1.33)$ & \\
\hline \multicolumn{6}{|c|}{ LTC4S } \\
\hline \multirow{3}{*}{ rs730012 } & AA & $234(68.8 \%)$ & $221(65.4 \%)$ & & \\
\hline & $\mathrm{AC}$ & $96(28.2 \%)$ & $104(30.8 \%)$ & $1.15(0.82-1.60)$ & 0.65 \\
\hline & $\mathrm{CC}$ & $10(2.9 \%)$ & $12(3.6 \%)$ & $1.27(0.54-3.00)$ & \\
\hline
\end{tabular}

years for controls, with age and gender being comparable across the two groups. Nearly $50.3 \%$ and $40.8 \%$ of cases were hypertensive and diabetic, respectively, which was significantly higher than the corresponding prevalence among controls $(14.4 \%$ and $15.9 \%$, resp. $)(P<0.001)$. There were 179 (54.2\%) myocardial infarction (MI) cases and 147 (44.5\%) non-MI cases. Frequency distribution of subjects based on the number of disease vessels was as follows: 0 or 1 vessel$59(18.4 \%), 2$ vessels- 83 (25.95\%), and 3 or more vessels-179 (55.8\%). TC and LDL-cholesterol levels were lower in CAD patients than in controls, which might be attributed to the higher usage of lipid lowering drugs in this group.

3.2. Assessment of Polymorphic Status in the Leukotriene Genes. We evaluated the polymorphic status of 136 functional SNPs by sequencing pooled DNA samples. While 91 markers were found to be invariant, the remaining 45 polymorphic SNPs were ranked to obtain a list of 14 functional SNPs with low to medium risk. 10 SNPs from literature were included and a total of 24 SNPs were genotyped in 680 subjects.

3.3. Association of Leukotriene SNPs with CAD. Except rs2660880, all other SNPs were in HWE in the controls $(P>$ $0.05)$. There was no significant difference in allele or genotype frequencies for any of the SNPs between cases and controls (Table 3). Also, there was no association between the SNPs and disease severity, based on the number of disease vessels. A SNP in the ALOX5 gene, rs12762303, showed significant association with CAD only in males, with the minor allele, "C" showing a protective effect $(\mathrm{OR} 0.31,95 \% \mathrm{CI}=0.12-0.81$; $P=0.007)$.

3.4. Haplotype Analysis. Figures S1(a)-S1(c) show the LD distribution among the Leukotriene SNPs of ALOX5, ALOX5AP, and LTA4H genes. There was strong pairwise LD between rs12762303 and rs1864414 SNPs in the ALOX5 gene $\left(r^{2}=\right.$ 0.99). rs4360791 and rs3803277 as well as rs9315050 and rs1132340 in ALOX5AP gene formed two distinct haplotype blocks. rs17222814 appeared to be the lead SNP in the ALOX5AP gene as it was in strong LD with the other SNPs in this gene $\left(r^{2}=0.90-0.96\right)$. There were two distinct haplotype blocks in the LTA4H gene, which included block 1 (5 SNPs) and block 2 (3 SNPs). Modest pair-wise LD was observed between rs2540475 and other SNPs in this gene $\left(r^{2}=0.41-0.73\right)$.

Distribution of haplotype frequencies is shown in Table S4. The TGAC haplotype in ALOX5 gene defined by 4 SNPs, rs12762303 rs2291427, rs1864414, rs41314980 and AGGGTCGCA haplotype in ALOX5AP gene defined 
by 9 SNPs, rs9315050, rs17216473, rs17222814, rs17222842, rs17222919, rs4769055 rs4360791, rs3803277, and rs1132340 and the TGATGTGCT haplotype in LTA4H gene constituted by 9 SNPs, rs17677715, rs2540475, rs2247570, rs6538697, rs61937881, rs2660898, rs2660899, rs17525495, and rs1978331 showed a frequency of $0.42,0.26$ and 0.32 in cases, respectively and were the best represented haplotypes in our cohort. However, none of the haplotypes showed significant association with CAD.

We also estimated the haplotype frequency of Hap A and Hap B of the ALOX5AP gene and compared them with the frequencies reported in the literature. Hap A comprised of rs172228149 (G), rs4769055(C), rs1132340(A), rs4360791(A) and Hap B comprised of rs17216473(A), rs4769055(A), and rs9315050(A), and rs17222842(G). Neither Hap A nor Hap $\mathrm{B}$ showed significant association with CAD. The distribution frequency is shown in Table S5.

3.5. Association of Plasma LTB4 Levels with CAD. Plasma LTB4 levels were measured in 150 cases and 150 matched controls. After removing the outliers, data on 141 cases and 144 controls was analyzed. LTB4 levels were $20 \%$ higher in cases as compared to controls $(76.42 \pm 4.46 \mathrm{pg} / \mathrm{mL}$ versus $60.89 \pm$ $2.61 \mathrm{pg} / \mathrm{mL} ; P=0.003$ ) (Figure 2). Four SNPs rs6538697, rs2660898, rs17525495, and rs1978331 in the LTA4H gene showed significant association with LTB4 levels (Figure 3(a)), with the minor allele being associated with lower LTB4 levels (Figure 3(b)). However, only one SNP, rs1978331 ( $P=0.035)$, retained significance following Bonferroni adjustment for multiple comparisons.

Plasma LTB4 levels were distributed into quartiles, Q1 (<37.59 pg/mL), Q2 (37.59-54.68 pg/mL), Q3 (54.69$85.59 \mathrm{pg} / \mathrm{mL})$, and Q4 (>85.59 pg/mL). Frequency of cases was higher in Q4 LTB4 levels $(n=43,30.5 \%)$ as compared to the controls $(n=28,19.4 \%)$. Subjects in the top LTB4 quartile showed significant higher risk of CAD as compared to the bottom quartile $(\mathrm{OR}=8.935,95 \% \mathrm{CI}=2.559-3.195$; $P=0.001$ ), after adjusting for potential confounders. Plasma LTB4 levels showed significant correlation with TC and LDLcholesterol $(r>0.30, P<0.0001)$, after adjusting for age, gender, and statins, particularly in cases but not in controls (Table S6).

\section{Discussion}

Leukotrienes are potent proinflammatory mediators belonging to the 5-lipoxygenase pathway, involved in Arachidonic acid metabolism. ALOX5, ALOX5AP, LTA4H, and LTC4S genes and their metabolic products have been investigated with regard to their role in the pathogenesis of cardiovascular disease [28]. In this pilot study, we examined the association of a select panel of Leukotriene SNPs encoded by these genes. In addition, we measured plasma LTB4 levels and tested its association with Leukotriene variants and CAD. Leukotriene SNPs did not show direct association with CAD but significant association was noted between plasma LTB4 levels and CAD as well as with four select SNPs in the LTA4H gene.

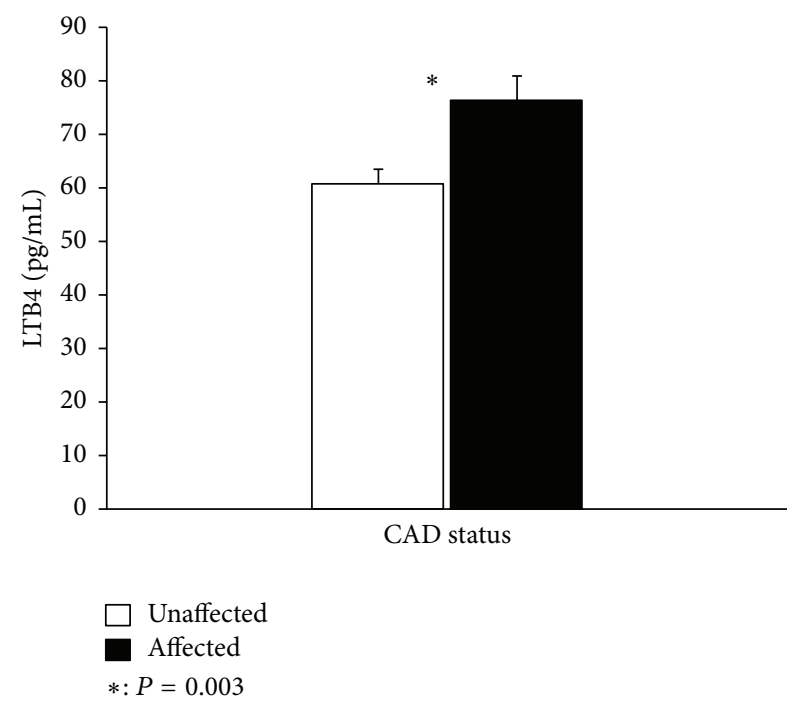

Figure 2: Distribution of mean LTB4 levels between affected and unaffected subjects. Data expressed as mean \pm standard deviation (SD).

The strength of our study lies in the use of a robust SNP selection process. Most of the genetic association studies carried out on Leukotriene gene variants have focused on markers without functional properties [11-13]. We have adopted a systematic process of selecting functional variants using in silico approach followed by targeted sequencing to establish their polymorphic status. In fact, reports suggest that it might be rewarding to study rare rather than common variants in order to understand the etiology of complex diseases [29]. Such rare variants were selected using SNPinfo and Genomatix tools along with the common variants found in the literature. To our knowledge, this is the first study to use a comprehensive in silico approach for SNP prioritization on the basis of functional relevance in the Leukotriene pathway. Of the 136 SNPs investigated, only 45 SNPs (30\%) were polymorphic, which could be attributed to a low minor allele frequency (MAF) of the rare functional variants in the Indian population. In fact, the average MAF was considerably higher for SNPs in the noncoding regions $(20 \%-25 \%)$ than in the coding regions $(12 \%-16 \%)$ [30], making it difficult to pick them up in our rather small-sized cohort. A recent review of GWAS suggests that more than $70 \%$ of genetic variants identified to date for CAD are located in the noncoding regions [31].

Family history is considered to be an independent risk factor for CAD [32]. Hence, in order to negate this bias, we included $225 \mathrm{CAD}$ subjects and 115 subjects with and without family history, respectively. None of the SNPs in either group showed significant association with the disease. The genetic contribution of Hap A and Hap B and their individual SNPs to the cardiovascular phenotypes in Whites, Blacks, and East Asian populations have yielded both positive $[11,12]$, and negative results $[17-19,33]$. In the Icelandic population, Hap A showed association with MI, with a case/control frequency of 0.158/0.095 [12], but no association in the Scottish $(0.184 / 0.142)$ [11] and Caucasian $(0.15 / 0.15)$ 


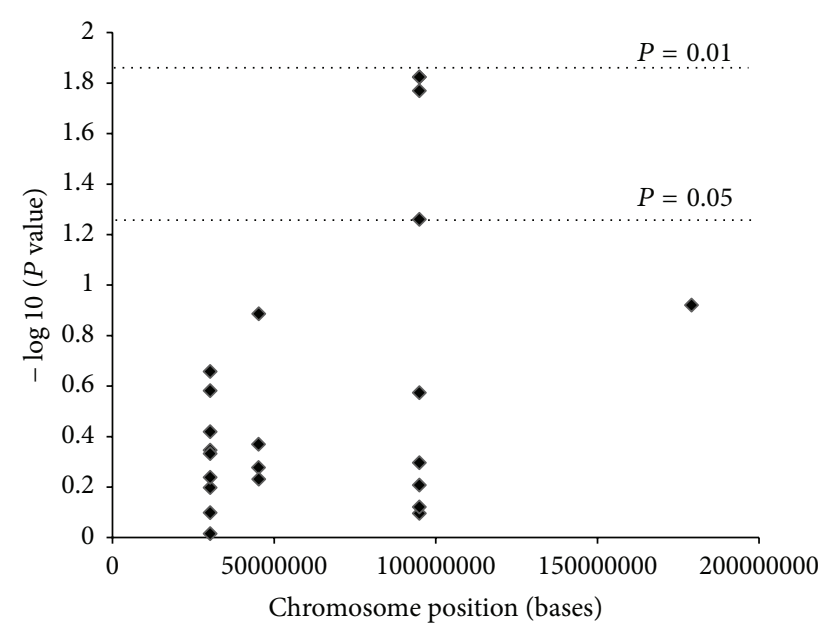

- SNPs

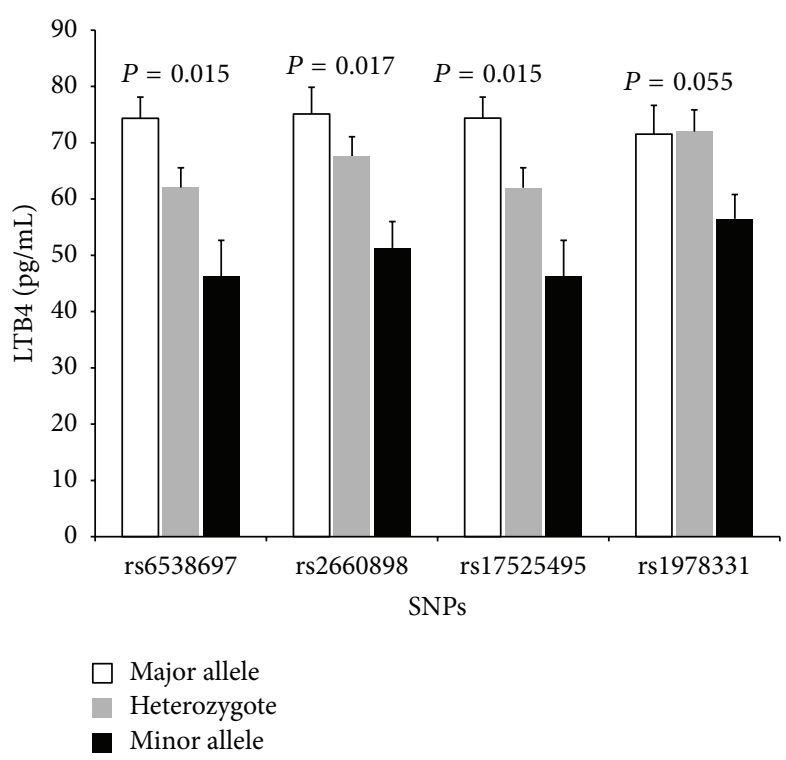

(b)

FIgURE 3: (a) Association results of all 24 SNPs with LTB4 are displayed. Genomic position ( $x$-axis) and negative log10 of $P$ values ( $y$-axis) are noted. SNPs meeting statistical significance have been annotated. (b) Bar diagram showing distribution of LTB4 in significant SNPs (rs6538697, rs2660898, rs17525495, and rs1978331) with different genotype. Bar diagram shows the distribution of LTB4 levels across the four LTA4H SNPs-rs6538697 (T>C), rs2660898 (T>G), rs17525495 (C>T), rs1978331 (T>C).

[34] populations. The case-control frequency obtained in our study $(0.13 / 0.15)$ was comparable to the German study (14.5/15.2) [18]. On the other hand, prevalence of Hap B in case controls was 0.07/0.07 in Iceland whites [12], 0.07/0.06 in Scottish whites [11], 0.09/0.08 in Caucasians [35], and 0.10/0.08 in African Americans [35]. The present study showed a frequency of $0.18 / 0.15$. None of the studies could demonstrate a significant association between Hap B and CAD. On the other hand, positive association was seen only in the Icelandic cohort [12], and this could be attributed to population-specific distribution in the allele/haplotype frequencies. Other factors such as phenotypic heterogeneity, age/gender bias, and, importantly, ethnic differences can also contribute to the genotype-phenotype association. Further, studies have dealt with varied phenotypes such as CAD, Myocardial Infarction (MI), and stroke, which makes comparisons and interpretations difficult.

The role of LTB4 levels in CAD pathogenesis may be mediated through the pro-inflammatory molecules such as IL-6, TNF- $\alpha$, and MCP-1 [36]. Previous studies have demonstrated increased LTB4 production in stimulated peripheral mononuclear cells in MI patients [12]. We observed higher LTB4 levels in cases than in controls and putative association between plasma LTB4 levels and 4 SNPs in the LTA4H gene. One of the SNPs, rs17525495, is in fact located in the promoter region and may modulate LTB4 production. The other 3 SNPs are located in the intronic region but exhibit strong LD with the promoter SNP which could explain their association with LTB4 levels. rs1978331 showed persistent association even after Bonferroni correction suggesting a robust association of this variant with plasma LTB4 levels. Thus, it appears that the LTA4H genetic variants regulate LTB4 production under the catalytic influence of the LTA4H enzyme. Evidence suggests that the rare T allele of rs17525495 increases LTA4H gene transcription [37], while the rare alleles of rs6538697, rs2660898, and rs1978331 are associated with lower LTB4 levels [13], which is comparable to the present study findings. Some studies have shown that LTB4 biosynthesis is regulated by cholesterol [38], and inhibition of Leukotriene may reduce plasma TG levels [39]. Our data also showed high correlation of LTB4 levels with TC, TG, and LDL-cholesterol, after adjusting for age, gender, and statins. Interestingly, this correlation was recorded in cases but not in controls reflecting the presence of an atherothrombotic state in CAD.

The short comings of the study are small sample size that might not be able to capture the true magnitude of the modest effect sizes of the genetic variants on CAD. Nonetheless, the interesting findings in this pilot study do provide a basis for planning larger studies on the Leukotriene pathway. Additionally, there may be sampling bias since the controls were selected based on their self-report of absence of any clinical symptoms and normal electrocardiogram readings which does not preclude the presence of a silent underlying disease. It has been demonstrated that $80 \%$ statistical power can be obtained under the assumption of 5\% prevalence, complete LD, 5\% $\alpha$ level given a sample size of 248 cases, and 248 controls for any particular SNP [40]. Under these circumstances, given a sample size of 340 cases and 340 controls and SNPs showing $>5 \%$ MAF, our study should have 
$>80 \%$ power to detect associations. It is however common knowledge that the power to detect small effect sizes of the alleles is duly enhanced in a larger cohort.

In conclusion, to our knowledge this is the first report of a case-control study designed to assess the association between functional polymorphisms in four key Leukotriene genes and CAD in a representative cohort of Asian Indians. Although there was no significant association between Leukotriene gene variants and disease, our preliminary finding showed higher LTB4 levels in CAD individuals and strong correlation between LTB4 levels and lipids in cases alone, reiterating the occurrence of an atherothrombotic state in these subjects. The association between LTA4H SNPs and plasma LTB4 levels suggests that these functional variants might modulate atherosclerosis by regulating LTB4 production. Future studies aimed at understanding the interactions between Leukotrienes and other key inflammatory biomarkers such as interleukins (IL 4, IL5, IL 6), TNF- $\alpha$, TGF- $\beta$, and IFN- $\gamma$ can throw better light on the inflammation-driven atherosclerotic disease process.

\section{Acknowledgments}

This work was supported by the Thrombosis Research Institute, London, the Department of Biotechnology, Ministry of Science and Technology, Government of India (Grant no. BT/01/CDE/08/07), Tata Social Welfare Trust, India (Grant no. TSWT/IG/SNB/JP/Sdm), the Weston foundation, and UK and Bey Foundation, Switzerland. The authors thank all the investigators, staff, administrative teams and participants of the IARS from Narayana Hrudayalaya, Bangalore, for their valuable contributions. The sponsors did not participate in the design, conduct, sample collection analysis and interpretation of the data or in the preparation, review, or approval of the paper. The authors have no conflicts of interests to disclose.

\section{References}

[1] S. Yusuf, S. Hawken, and S. Ounpuu, "Effect of potentially modifiable risk factors associated with myocardial infarction in 52 countries (the INTERHEART study): case-control study," The Lancet, vol. 364, no. 9450, pp. 937-952, 2004.

[2] D. Steinberg, "Atherogenesis in perspective: hypercholesterolemia and inflammation as partners in crime," Nature Medicine, vol. 8, no. 11, pp. 1211-1217, 2002.

[3] M. Peters-Golden and W. R. Henderson Jr., "Leukotrienes," The New England Journal of Medicine, vol. 357, no. 18, pp. 1841-1854, 2007.

[4] C. D. Sadik and A. D. Luster, "Lipid-cytokine-chemokine cascades orchestrate leukocyte recruitment in inflammation," Journal of Leukocyte Biology, vol. 91, no. 2, pp. 207-215, 2012.

[5] M. Bäck and G. K. Hansson, "Leukotriene receptors in atherosclerosis," Annals of Medicine, vol. 38, no. 7, pp. 493-502, 2006.

[6] M. Mehrabian and H. Allayee, "5-Lipoxygenase and atherosclerosis," Current Opinion in Lipidology, vol. 14, no. 5, pp. 447-457, 2003.
[7] G. Riccioni, A. Zanasi, N. Vitulano, B. Mancini, and N. D'Orazio, "Leukotrienes in atherosclerosis: new target insights and future therapy perspectives," Mediators of Inflammation, vol. 2009, Article ID 737282, 6 pages, 2009.

[8] A. Di Gennaro, D. Wagsáter, M. I. Máyránpáá et al., "Increased expression of leukotriene C4 synthase and predominant formation of cysteinyl-leukotrienes in human abdominal aortic aneurysm," Proceedings of the National Academy of Sciences of the United States of America, vol. 107, no. 49, pp. 21093-21097, 2010.

[9] R. Spanbroek, R. Gräbner, K. Lötzer et al., "Expanding expression of the 5-lipoxygenase pathway within the arterial wall during human atherogenesis," Proceedings of the National Academy of Sciences of the United States of America, vol. 100, no. 3, pp. 1238-1243, 2003.

[10] H. Qiu, A. Gabrielsen, H. E. Agardh et al., "Expression of 5-lipoxygenase and leukotriene A4 hydrolase in human atherosclerotic lesions correlates with symptoms of plaque instability," Proceedings of the National Academy of Sciences of the United States of America, vol. 103, no. 21, pp. 8161-8166, 2006.

[11] A. Helgadottir, S. Gretarsdottir, D. St. Clair et al., "Association between the gene encoding 5-lipoxygenase-activating protein and stroke replicated in a Scottish population," American Journal of Human Genetics, vol. 76, no. 3, pp. 505-509, 2005.

[12] A. Helgadottir, A. Manolescu, G. Thorleifsson et al., "The gene encoding 5-lipoxygenase activating protein confers risk of myocardial infarction and stroke," Nature Genetics, vol. 36, no. 3, pp. 233-239, 2004.

[13] A. Helgadottir, A. Manolescu, A. Helgason et al., "A variant of the gene encoding leukotriene A4 hydrolase confers ethnicityspecific risk of myocardial infarction," Nature Genetics, vol. 38, no. 1, pp. 68-74, 2006.

[14] H. Allayee, A. Baylin, J. Hartiala et al., "Nutrigenetic association of the 5-Lipoxygenase gene with myocardial infarction," American Journal of Clinical Nutrition, vol. 88, no. 4, pp. 934-940, 2008.

[15] J. H. Dwyer, H. Allayee, K. M. Dwyer et al., "Arachidonate 5lipoxygenase promoter genotype, dietary arachidonic acid, and atherosclerosis," The New England Journal of Medicine, vol. 350, no. 1, pp. 29-37, 2004.

[16] D. M. Iovannisci, E. J. Lammer, L. Steiner et al., "Association between a leukotriene C4 synthase gene promoter polymorphism and coronary artery calcium in young women: the Muscatine study," Arteriosclerosis, Thrombosis, and Vascular Biology, vol. 27, no. 2, pp. 394-399, 2007.

[17] W. Koch, P. Hoppmann, J. C. Mueller, A. Schömig, and A. Kastrati, "No association of polymorphisms in the gene encoding 5-lipoxygenase-activating protein and myocardial infarction in a large central European population," Genetics in Medicine, vol. 9, no. 3, p. 187, 2007.

[18] E. Lõhmussaar, A. Gschwendtner, J. C. Mueller et al., "ALOX5AP gene and the PDE4D gene in a central European population of stroke patients," Stroke, vol. 36, no. 4, pp. 731-736, 2005.

[19] R. Y. L. Zee, S. Cheng, H. H. Hegener, H. A. Erlich, and P. M. Ridker, "Genetic variants of arachidonate 5-lipoxygenaseactivating protein, and risk of incident myocardial infarction and ischemic stroke: a nested case-control approach," Stroke, vol. 37, no. 8, pp. 2007-2011, 2006.

[20] J. Shanker, A. Maitra, V. S. Rao et al., "Rationale, design and preliminary findings of the Indian atherosclerosis research study," Indian Heart Journal, vol. 62, no. 4, pp. 286-295, 2010. 
[21] N. K. Kumar, "Bioethics activities in India," Eastern Mediterranean Health Journal, vol. 12, no. 1, pp. S56-S65, 2006.

[22] W. T. Friedewald, R. I. Levy, and D. S. Fredrickson, "Estimation of the concentration of low-density lipoprotein cholesterol in plasma, without use of the preparative ultracentrifuge," Clinical Chemistry, vol. 18, no. 6, pp. 499-502, 1972.

[23] Z. Xu and J. A. Taylor, "SNPinfo: integrating GWAS and candidate gene information into functional SNP selection for genetic association studies," Nucleic Acids Research, vol. 37, no. 2, pp. W600-W605, 2009.

[24] H. Yuan, J. Chiou, W. Tseng et al., "FASTSNP: an always upto-date and extendable service for SNP function analysis and prioritization," Nucleic Acids Research, vol. 34, pp. W635-W641, 2006.

[25] S. A. Miller, D. D. Dykes, and H. F. Polesky, "A simple salting out procedure for extracting DNA from human nucleated cells," Nucleic Acids Research, vol. 16, no. 3, article 1215, 1988.

[26] P. Sham, J. S. Bader, I. Craig, M. O’Donovan, and M. Owen, "DNA pooling: a tool for large-scale association studies," Nature Reviews Genetics, vol. 3, no. 11, pp. 862-871, 2002.

[27] J. Wang, K. Chuang, M. Ahluwalia et al., "High-throughput SNP genotyping by single-tube PCR with Tm-shift primers," BioTechniques, vol. 39, no. 6, pp. 885-892, 2005.

[28] B. Haribabu, "Leukotrienes: novel targets for vascular disease," Discovery Medicine, vol. 4, pp. 281-287, 2004.

[29] W. Bodmer and C. Bonilla, "Common and rare variants in multifactorial susceptibility to common diseases," Nature Genetics, vol. 40, no. 6, pp. 695-701, 2008.

[30] K. A. B. Goddard, P. J. Hopkins, J. M. Hall, and J. S. Witte, "Linkage disequilibrium and allele-frequency distributions for 114 single-nucleotide polymorphisms five populations," American Journal of Human Genetics, vol. 66, no. 1, pp. 216-234, 2000.

[31] R. Roberts and A. F. R. Stewart, "9p21 and the genetic revolution for coronary artery disease," Clinical Chemistry, vol. 58, no. 1, pp. 104-112, 2012.

[32] S. Shea, R. Ottman, and C. Gabrieli, "Family history as an independent risk factor for coronary artery disease," Journal of the American College of Cardiology, vol. 4, no. 4, pp. 793-801, 1984.

[33] G. Wang, Y. Wang, H. Sun et al., "Variants of the arachidonate 5-lipoxygenase-activating protein (ALOX5AP) gene and risk of ischemic stroke in Han Chinese of eastern China," Journal of Biomedical Research, vol. 25, no. 5, pp. 319-327, 2011.

[34] J. Hartiala, D. Li, D. V. Conti et al., "Genetic contribution of the leukotriene pathway to coronary artery disease," Human Genetics, vol. 129, no. 6, pp. 617-627, 2011.

[35] D. R. Crosslin, S. H. Shah, S. C. Nelson et al., "Genetic effects in the leukotriene biosynthesis pathway and association with atherosclerosis," Human Genetics, vol. 125, no. 2, pp. 217-229, 2009.

[36] F. Stanke-Labesque, J. L. Pepin, T. de Jouvencel, C. Arnaud, J. P. Baguet et al., "Leukotriene B4 pathway activation and atherosclerosis in obstructive sleep apnea," Journal of Lipid Research, vol. 53, pp. 1944-1951, 2012.

[37] D. M. Tobin, F. J. Roca, S. F. Oh et al., "Host genotypespecific therapies can optimize the inflammatory response to mycobacterial infections," Cell, vol. 148, no. 3, pp. 434-446, 2012.

[38] A. N. Zagryagskaya, D. A. Aleksandrov, M. A. Pushkareva, S. I. Galkina, Z. V. Grishina, and G. F. Sud'Ina, "Biosynthesis of leukotriene B4 in human polymorphonuclear leukocytes: regulation by cholesterol and other lipids," Journal of Immunotoxicology, vol. 5, no. 4, pp. 347-352, 2008.

[39] J. Choi, H. J. Jeon, J. Park et al., "Anti-atherogenic effect of BHBTZD having inhibitory activities on cyclooxygenase and 5lipoxygenase in hyperlipidemic mice," Atherosclerosis, vol. 212, no. 1, pp. 146-152, 2010.

[40] E. P. Hong and J. W. Park, "Sample size and statistical power calculation in genetic association studies," Genomics \& Informatics, vol. 10, pp. 117-122, 2012. 


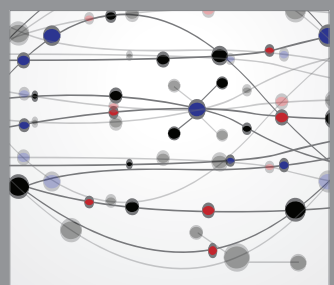

The Scientific World Journal
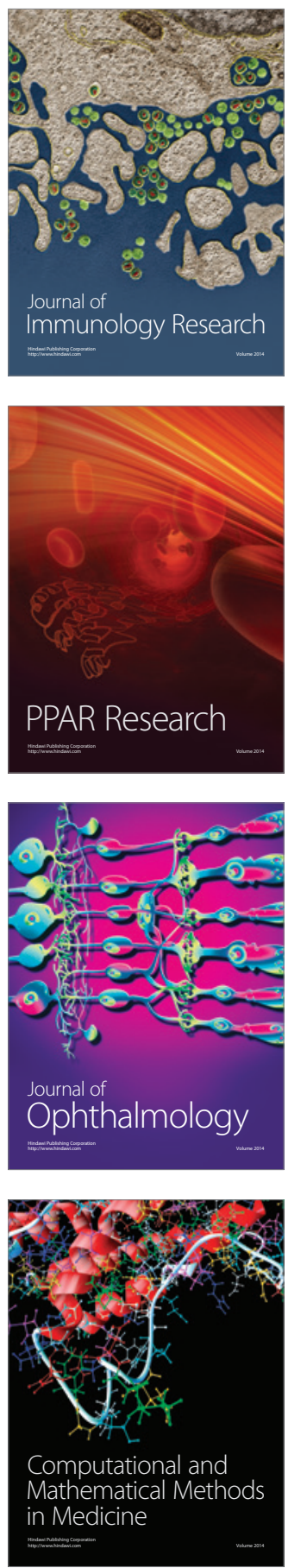

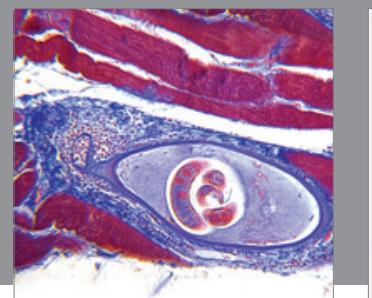

Gastroenterology

Research and Practice
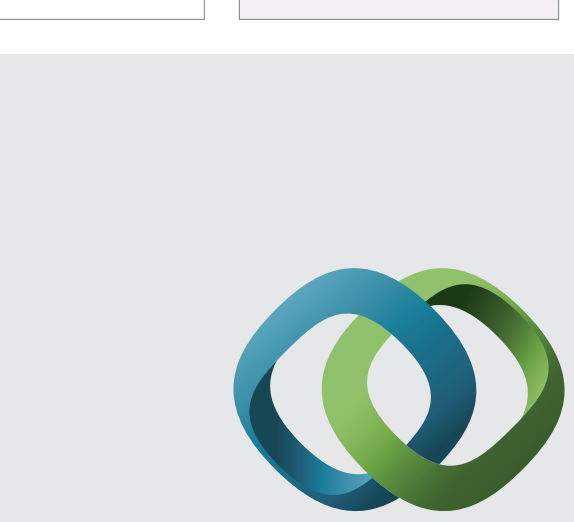

\section{Hindawi}

Submit your manuscripts at

http://www.hindawi.com
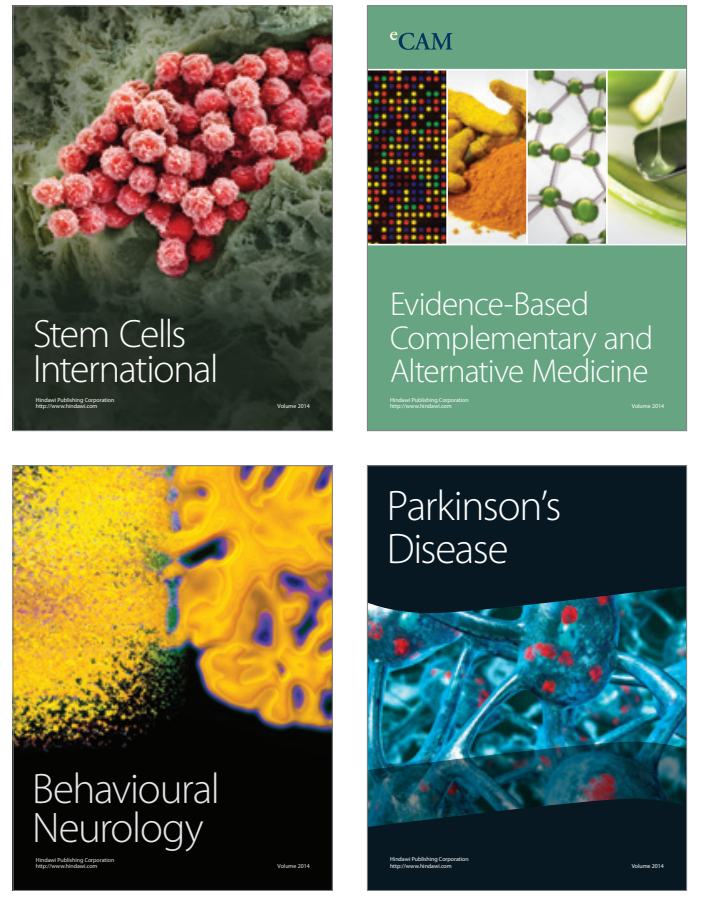
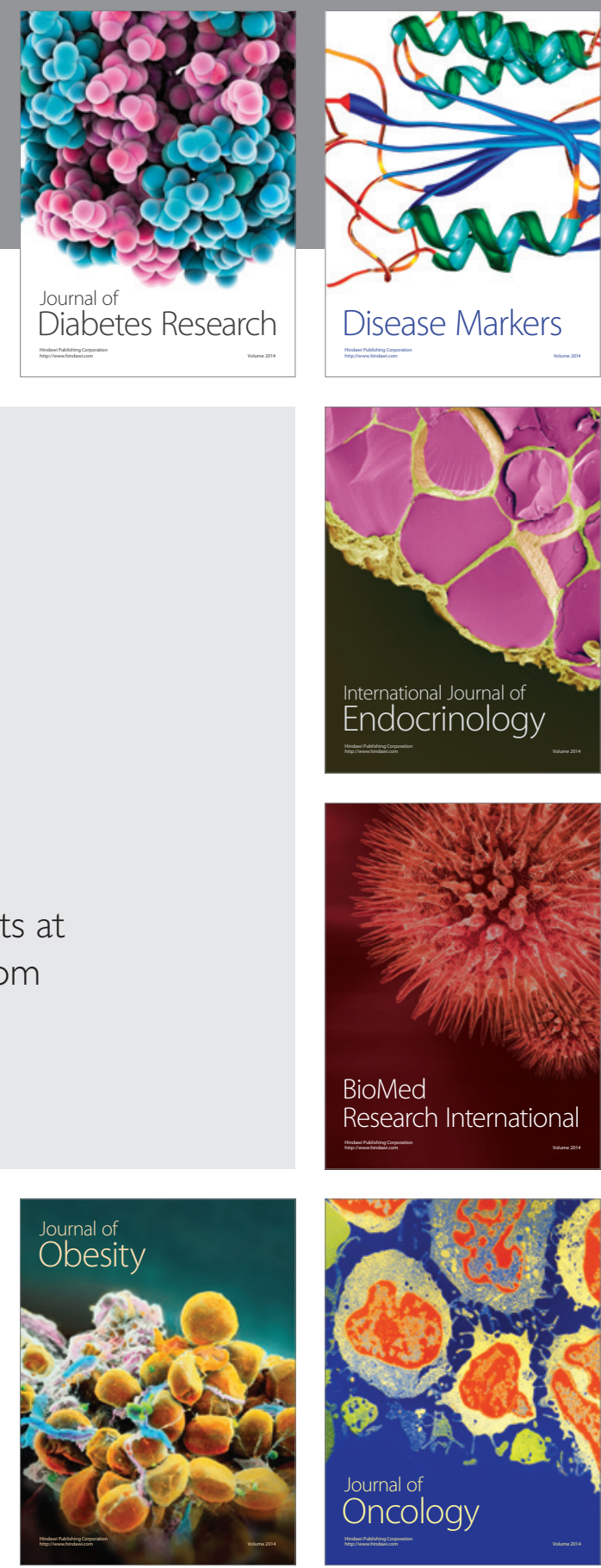

Disease Markers
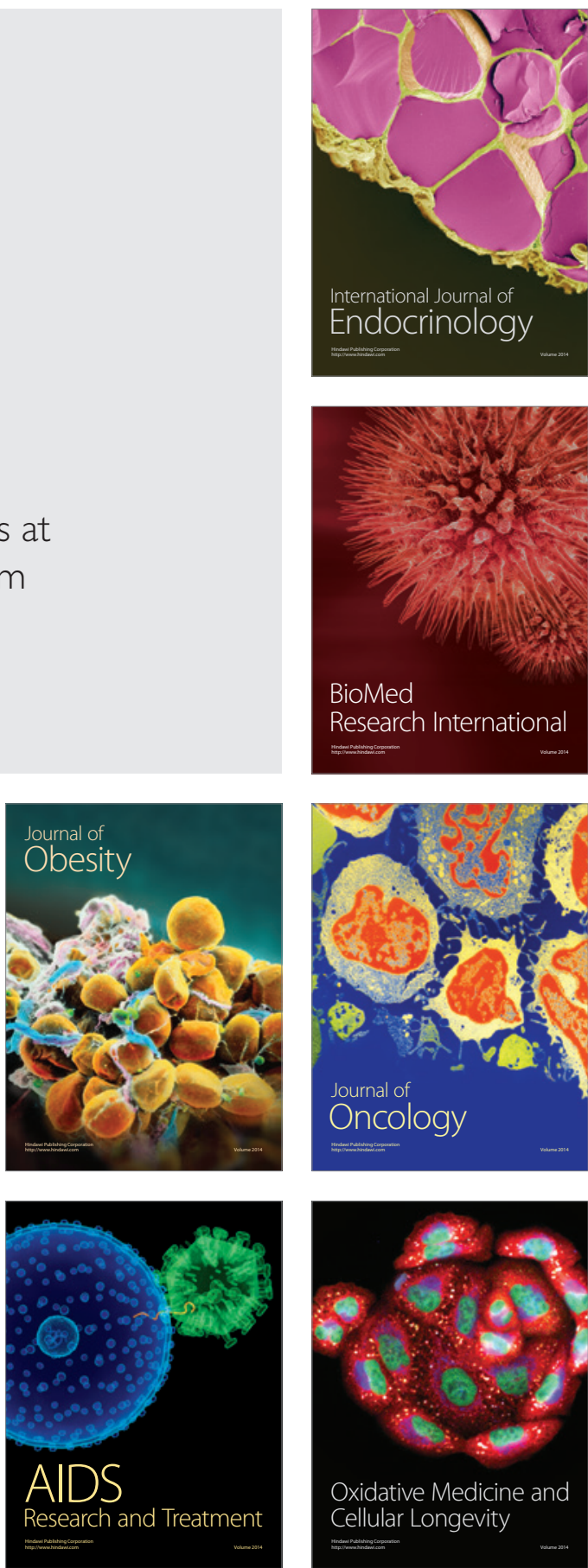